\title{
Bodenentwicklung im Hochgebirge: Ein Beispiel vom Vorfeld des Steingletschers in der Schweiz
}

Beim Zurückschmelzen eines Gletschers werden die entblößten subglazialen Ablagerungen sukzessiv den bodenbildenden Prozessen ausgesetzt. In einem solchen Bereich ergeben sich gute Möglichkeiten, die Bodenentwicklung zu studieren, besonders wenn die Deglaziationschronologie durch glazialmorphologische Untersuchungen festgelegt ist. Eine solche bestimmte Altersabfolge war im Bereich des ehemaligen Vergletscherungsgebietes des Steingletschers in der zentralen Schweiz gegeben, wo stichprobenartig drei Podsolprofile auf Moränen verschiedenen Alters untersucht wurden. Zweck der Untersuchung war, den Einfluß sowohl der naturbedingten Prozesse als auch der Einwirkung des Menschen zu verfolgen.

\section{Das Untersuchungsgebiet}

Die Lage des Steingletschergebietes geht aus Abb. 1 hervor. Die postglaziale Gletscher- und Vegetationsgeschichte des Gebietes ist in jüngster Zeit von KING (1974) studiert worden. Dabei sind auch die Altersverhältnisse der Eisrandlagen erläutert worden. KING hat auch einige Bodenprofile beschrieben, aber mit Ausnahme einiger Pollenanalysen nicht näher analysiert. Das Gebiet liegt oberhalb der Waldgrenze; nach KING (1974, S. 14 und 15) liegt die natürliche Waldgrenze bei etwa $1900 \mathrm{~m}$, die heutige Waldgrenze infolge Tätigkeit des Menschen aber 100 bis $200 \mathrm{~m}$ tiefer. Nur unsere niedrigste Bodenbeobachtungsstelle (Punkt 1 in Abb. 1) liegt auf der Grenze zur subalpinen Nadelwaldstufe.

\section{Gang der Untersuchung}

Die Untersuchung wurde (neben der Bearbeitung anderer Probleme) während einer Studienexkursion des Geographischen Instituts der Universität Helsinki im Juni 1978 durchgeführt. Drei Bodenprofile (1, 2 und 3 in Abb. 1) wurden aufgeschlossen, gemessen, beschrieben und Materialproben entnommen. Die Proben wurden nachher im Labor der Firma Viljavuuspalvelu $\mathrm{Oy}$ in Helsinki analysiert mit Bezug auf $\mathrm{pH}$, Humusgehalt und Gehalt an Schwefel, Eisen, Aluminium und Silizium. Granulometrische Bestimmungen wurden im Geologischen Institut der
Universität Helsinki gemacht. Im Gelände wurde auch eine Übersichtskartierung der Vegetation vorgenommen.

\section{Bodenbildende Faktoren im Studiengebiet}

\section{Die Zeit}

Problem dieser Untersuchung ist vor allem festzustellen, innerhalb welcher Zeit die Podsole im Gebirge sich so weit entwickeln, daß man die Bodenhorizonte zum mindesten mit chemischen Analysen nachweisen kann. Beobachtungspunkt 1 wurde vor schon fast 10000 Jahren vom Steingletscher freigegeben (KING 1974, S. 63 u. 68) und das dortige Profil deutet darauf hin, wie sich der Boden im Ablauf einer längeren Zeit entwickeln kann. Die Punkte 2 und 3 sind auf jüngerem Untergrund gelegen; Punkt 2 auf einem vom vorstoßenden Steingletscher im 17. Jahrhundert aufgeschütteten Stirnmoränenwall und Punkt 3 auf einer Stirnmoräne vom Gletschervorstoß in den Jahren 1850-1860 (s. Abb. 1; vgl. KING 1974). Die Wirkungszeit der bodenbildenden Prozesse beträgt im Punkt 2 somit 300-350 Jahre und im Punkt 3 nur 120-130 Jahre.

\section{Die Topographie}

Mit Hinsicht auf die Großformen liegt das Untersuchungsgebiet in einem von Bergen umgebenen Tal, vor Winden, jedoch nicht vor den vom Gletscher katabatisch niederfließenden Kaltluftmassen geschützt. Punkt 1 in einer Höhe von etwas über $1800 \mathrm{~m}$ liegt mikrotopographisch auf einer flachen südgeneigten Hangfazette unterhalb einer steilen Böschung. In dieser Lage hält sich der Boden feucht, und das Temperaturmikroklima ist vorteilhaft. Die Probestellen 2 und 3 liegen beide auf den Südhängen in der Nähe der Scheitel der entsprechenden Moränenwälle, in einer Höhe von über $1900 \mathrm{~m}$.

Olavi Heikkinen and Paul Fogelberg, Department of Geography, University of Helsinki, Hallituskatu 11-13, SF-00100 Helsinki 10, Finland. 


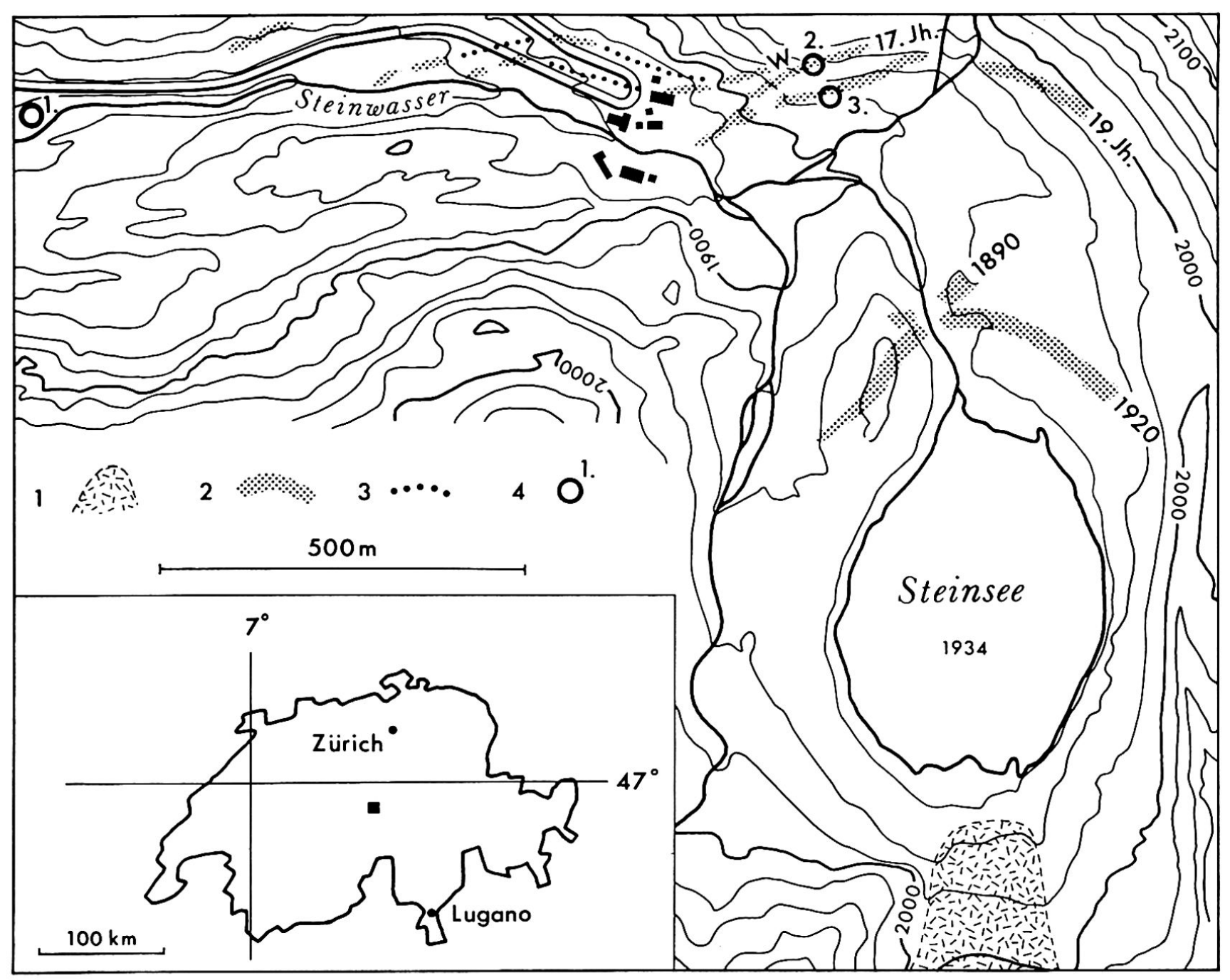

Abb. 1 Das Untersuchungsgebiet. Die Moränenwälle und deren Altersdeutung nach KING (1974). Die Topographie basiert auf der Landeskarte der Schweiz 1:25000, Blatt 1211 Meiental. Äquidistanz der Höhenlinien 20 m. (1) Steingletscher (die Lage des Gletscherrandes ist nur ungenau eingezeichnet). (2) Moränenwälle mit Entstehungszeitpunkt. (3) Verbindungslinie Eisrandlagen. (4) Probenentnahmestelle mit Nummer. Die Gebäude an der Haarnadelkurve der Sustenpaßstraße (oben in der Karte) gehören zum Hotel Steingletscher.

\section{Das Ausgangsmaterial}

Das Muttergestein spielt eine entscheidende Rolle besonders im Anfang der Bodenentwicklung. Der Felsgrund im Steingletschergebiet besteht hauptsächlich aus kristallinen Gesteinen: Granit und Gneis (sPICHER 1972). Somit ist auch das vom Gletscher aufgeschüttete Moränenmaterial vorwiegend sauer und silikatreich. Dieses nährstoffarme Substrat begünstigt die Entwicklung von Podsolböden.

Den chemischen Analysen der Bodenprofile (Abb. 5, 6 und 7) entsprechen granulometrische Analysen (Abb. 2, 3 und 4) aus denselben Tiefen. Die Buchstaben A, B und C im Anschluß an die Korngrößenkurven entsprechen entweder den deutlich sichtbaren (Profil 1) oder den zum Teil vermuteten (Profilen 2 und 3) A-, B- und C-Bodenhorizonten.

Im alten Bodenprofil (Abb. 2) fällt der Medianwert des Materials in die Korngrößengruppe Grobsand

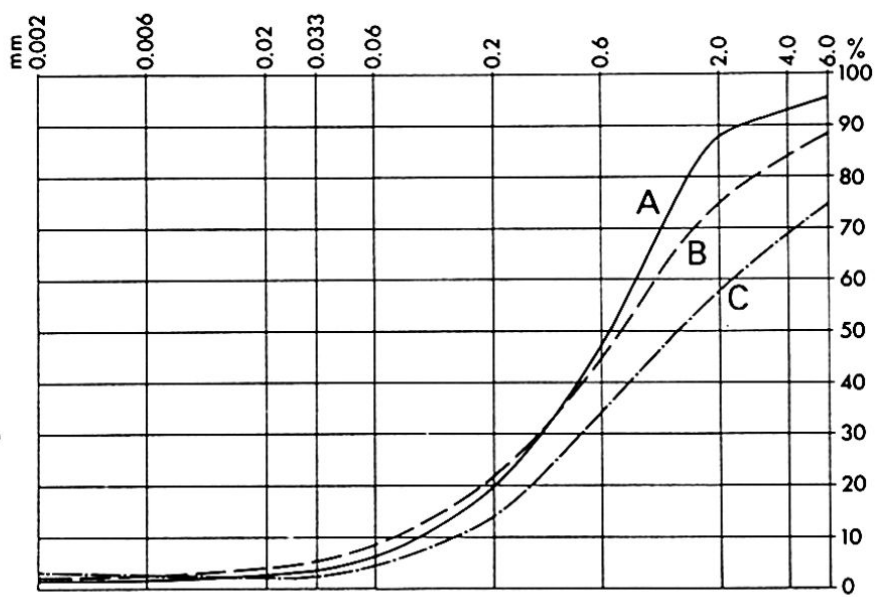

Abb. 2 Korngrößenverteilung im Bodenprofil 1, A-, B- und C-Horizonte. Die Zahl $100 \%$ bezieht sich auf Material mit einer Korngröße weniger als 20 mm; das gröbere Material wurde bei der Probenentnahme ausgeschieden. 


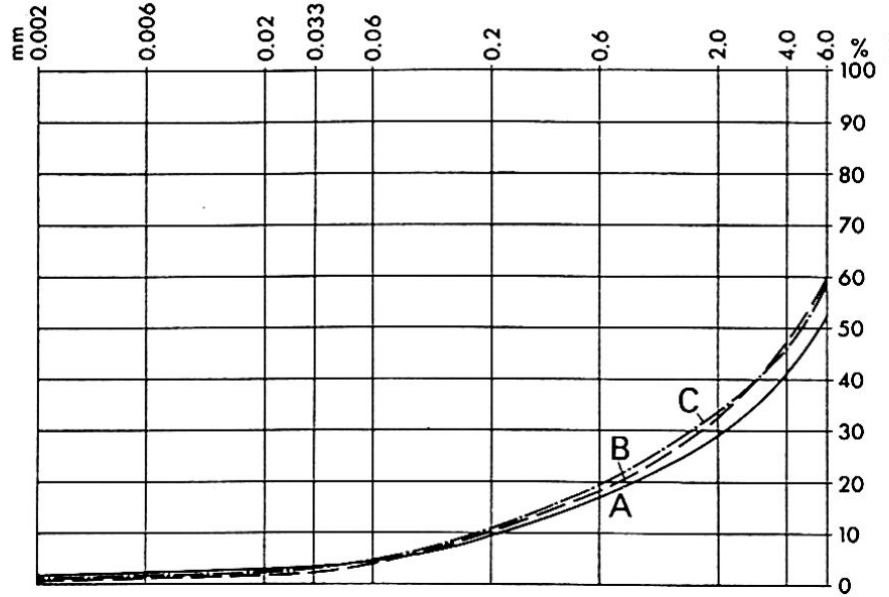

Abb. 3. Korngrößenverteilung im Bodenprofil 2. S. Bemerkungen wie bei Abb. 2.

(Klassifikation ATTERBERG; KÖSTER \& LESER 1967,S. 93), und das Material kann als Sandmoräne bezeichnet werden. Besonders das Oberflächenmaterial ist deutlich sortiert, anscheinend infolge Materialtransport durch die Schmelzwässer am Ende oder nach der Deglaziation. Der Boden im eigentlichen Sinn, die A- und B-Horizonte, enthält nur wenig Steine und Blöcke. Dagegen ist das Material der Profile 2 und 3 (Abb. 3 und 4) durchgehend Kiesmoräne mit reichlich Steinen und Blöcken. Das Material sämtlicher Profile ist gut wasserdurchlässig, was die der Podsolierung zugehörige Auswaschung der oberflächennahen Schichten fördert.

\section{Das Klima}

Es ist gut bekannt, daß besonders die Feuchtigkeitsund Temperaturverhältnisse die Entwicklungsrichtung des Bodens regulieren. Die durchschnittliche jährliche Niederschlagssumme, gemessen an der nahe gelegenen Wetterstation Grimsel (Höhe 1956 m ü. M., was etwa dem Untersuchungsgebiet entspricht), beträgt $2150 \mathrm{~mm}$. Der Niederschlag verteilt sich ziemlich gleichmäßig auf die Monate des Jahres (UTTINGER 1965). Schnee fällt im Untersuchungsgebiet gewöhnlich auch in den Sommermonaten (KING 1974, S. 15). Jahresmitteltemperatur in Grimsel ist $1,2^{\circ} \mathrm{C}$. Mitteltemperatur des wärmsten Monats, Juli, ist $9,1^{\circ} \mathrm{C}$ (sCHÜEPP 1960). Die kurzen, kühlen Sommer, die reichlichen Schmelzwässer im Frühling und die verhältnismäßig hohen Niederschläge im Sommer bedeuten als Ganzes ein feuchtes, kühles Klima, was die Podsolierung des Bodens fördert. Im Vorfeld des Steingletschers ist Podsol der häufigste Bodentyp, aber auch Braunerde kommt vor (KING 1974).

\section{Die Vegetation}

Die Vegetation, von den oben behandelten Faktoren bedingt, befestigt die Bodenoberfläche und beeinflußt

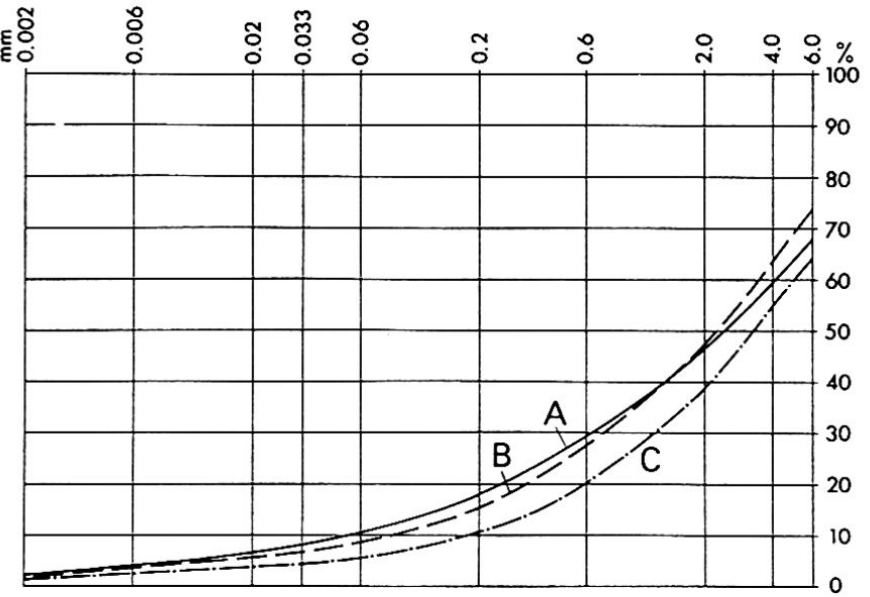

Abb. 4. Korngrößenverteilung im Bodenprofil 3. S. Bemerkungen wie bei Abb. 2 .

kräftig insbesondere die Humusbildung im Boden. Es mag bis über 3000 Jahre dauern, ehe die völlig kahlen Moränenschuttgebiete im Hochgebirge eine geschlossene Klimaxvegetationsdecke erhalten (zoLLITSCH 1969). Trotzdem ist die Feldschicht der Vegetation, die hauptsächlich von Kräutern und Zwergsträuchern gebildet wird, ziemlich einheitlich, sowohl im Bereich des ältesten Bodens an der Baumgrenze, die dort von Bergföhren gebildet wird, als auch im Bereich der zwei jüngeren Bodenprofile an den Moränenwällen in der baumlosen alpinen Stufe. Die Vegetationsdecke der Moränenwälle vom 17. Jahrhundert und von den 1850er Jahren zeigt aber große Variationen. Stellenweise ist der Boden fast kahl infolge von Periglazialprozessen oder Abtragung durch Beweidung. Andererseits sind Stellen mit feinmaterialreichem Moränenschutt sowie die vom Vieh gedüngten Flächen stark bewachsen.

\section{Bodenprofile und chemische Analysen}

\section{Profil 1}

Die Einflußdauer der bodenbildenden Prozesse beträgt hier schon fast $10000 \mathrm{Jahre}$. In Abb. 5 sind die auch mit blossem Auge deutlich sichtbaren Horizonte sowie ihre Farben den Munsell Soil Color Charts (1954) gemäß dargestellt. Die Abbildung zeigt auch die aus den drei Horizonten gemessenen Totalmengen $(\mathrm{mg} / \mathrm{g})$ von Schwefel, Eisen, Aluminium und Silizium, die Prozentanteile des Humusgehaltes und die $\mathrm{pH}$-Werte. Die Mächtigkeit des organischen Horizonts $\left(\mathrm{A}_{0}\right)$ an der Oberfläche beträgt ca. $4 \mathrm{~cm}$. Er wird von einem $8 \mathrm{~cm}$ mächtigen leichtgrauen Bleichhorizont $\left(\mathrm{A}_{\mathrm{e}}\right)$ unterlagert. Der Bleichhorizont bildet einen wesentlichen Teil des Eluvial- oder Auswaschungshorizonts und ist für den Podsol charakteristisch. Unterhalb des Eluvialhorizonts liegt ein $13 \mathrm{~cm}$ mächtiger, aus zwei Subhorizonten bestehender Illuvial- oder Anreiche- 


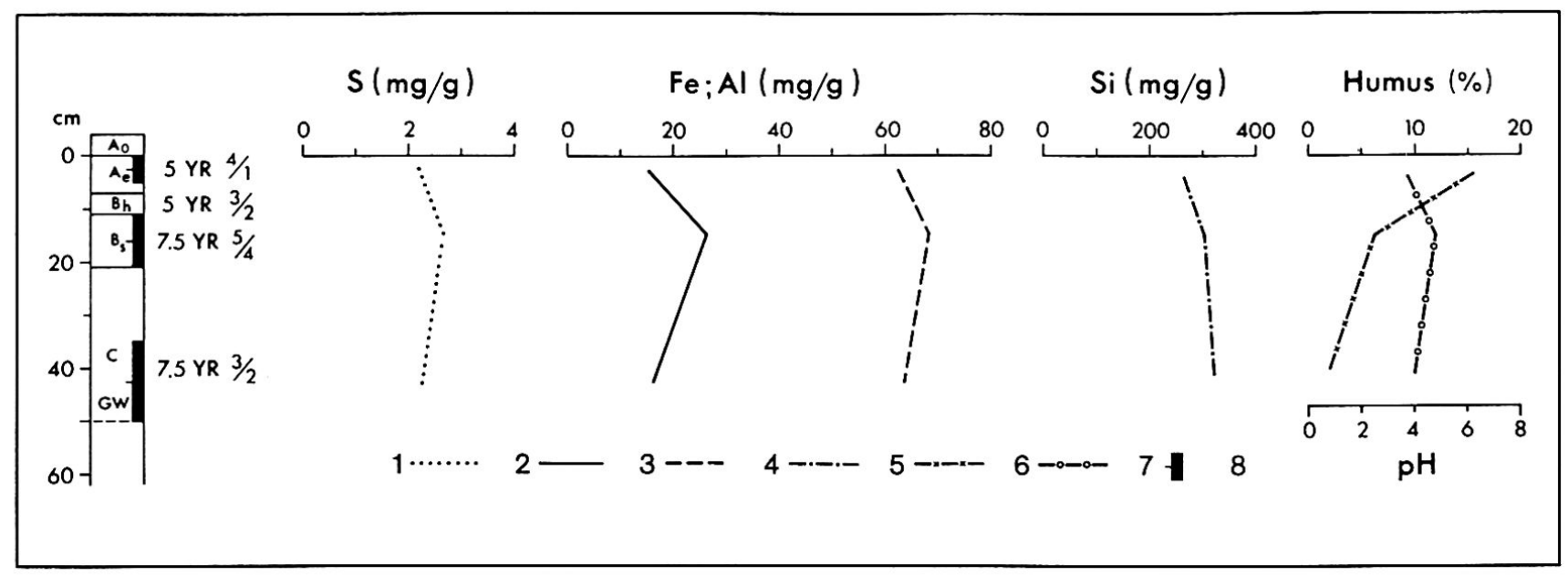

Abb. 5. Chemische Analysen. Bodenprofil 1 (Alter fast 10000 Jahre). Erläuterung der Symbole: (1) Schwefel, (2) Eisen, (3) Aluminium, (4) Silizium, (5) Humus, (6) pH, (7) Probe für Analyse, (8) Grundwasserspiegel.

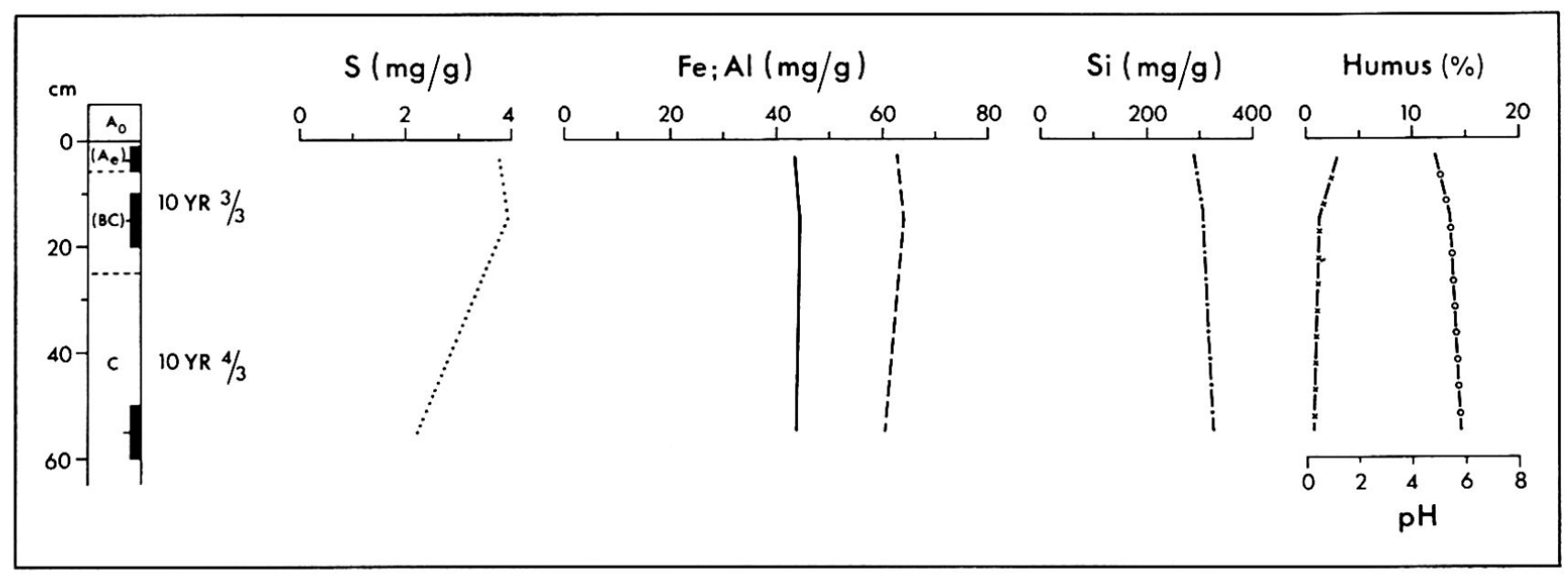

Abb. 6. Chemische Analysen, Bodenprofil 2 (Alter 300-350 Jahre). Symbole wie in Abb. 5.

rungshorizont. Der obere Subhorizont ist eine durch Humusniederschlag dunkelgefärbte Humusorterde $\left(B_{h}\right)$ mit lockerer Struktur. Der untere Subhorizont ist eine rostbraune Eisenorterde $\left(B_{s}\right)$. Der Illuvialhorizont geht allmählich in unveränderten Untergrund über (C). Zur Zeit der Probeentnahme, am 13. Juni 1978, lag der Grundwasserspiegel in $50 \mathrm{~cm}$ Tiefe. - Der Bodentyp im Profil 1 kann als Eisenhumuspodsol bezeichnet werden.

Die chemischen Analysen aus den Horizonten $\mathrm{A}_{e}, \mathrm{~B}_{\mathrm{s}}$ und $C$ zeigen, $\mathrm{da} ß$ besonders Eisen und Aluminium aus dem Eluvialhorizont ausgelaugt und in den Illuvialhorizont als Eisentonerde angereichert worden sind. Die Auslaugung des $\mathrm{A}_{\mathrm{e}}$-Horizonts hat den pH-Wert dort erniedrigt. Bei Silizium ist der Trend nicht so deutlich wie bei Eisen und Aluminium. Auslaugung von Silizium ist auch kein dem Podsolboden kennzeichnender Prozeß (sCHEFFER u. SCHACHTSCHABEL 1970, S. 334 bis 336). Der Anteil des Humus kann als überraschend groß bezeichnet werden, wird aber dadurch erklärt, daß die Pflanzenwurzeln tief in den Boden eindringen und da $ß$ der Humusgehalt überhaupt mit der Zeit im Boden wächst (BURGER u. FRANZ 1969, S. 261).

\section{Profil 2}

Profil 2 stammt von einem Moränenwall vom 17. Jahrhundert. Die obersten Teile des Profils werden von einer hauptsächlich organogenen, dunklen, $7 \mathrm{~cm}$ dicken Schicht gebildet, die $\mathrm{A}_{0}$-Horizont genannt worden ist (Abb. 6), obwohl sie auch Mineralboden enthält. Eigentliche Horizonte im Mineralboden sind mit dem Auge schwer zu unterscheiden. Zwar unterscheidet sich die braune Farbe der Oberflächenschicht von den liegenden Schichten. Wir nehmen an, daß die Farbänderung in $25 \mathrm{~cm}$ Tiefe die obere Abgrenzung des unveränderten Untergrunds (C) darstellt. Die chemischen Analysen bestätigen diesen Befund. Der Eluvialhorizont, der $\mathrm{A}_{\mathrm{e}}$-Horizont in Abb. 6, war nicht mit bloßem Auge zu sehen, sondern die Abgrenzung basiert auf den Ergebnissen der chemischen Analysen. Die chemischen Unterschiede in diesem Profil sind 


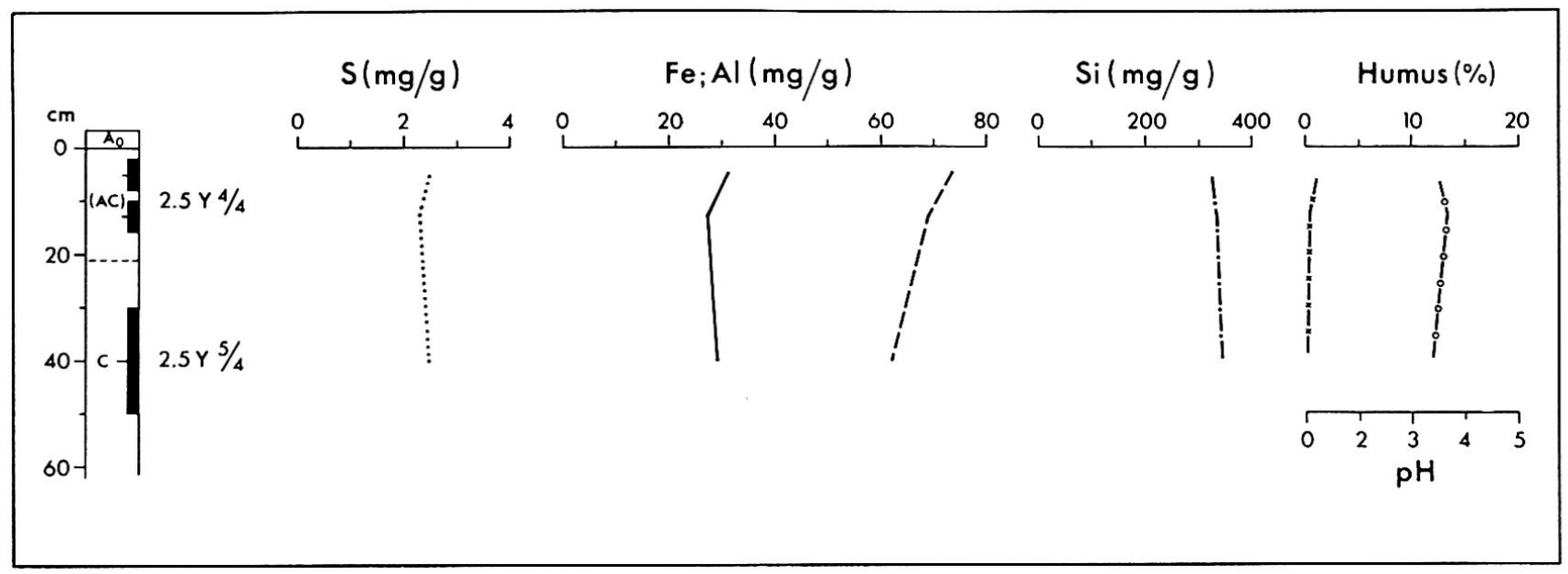

Abb. 7. Chemische Analysen, Bodenprofil 3 (Alter 120-130 Jahre). Symbole wie in Abb. 5.

gering. Trotzdem deuten die Kurven in Abb. 6 jedoch widerspruchslos eine Podsolierung an.

\section{Profil 3}

Die Probeentnahmestelle befindet sich an einem in den 1850er Jahren aufgeschütteten Moränenwall. Unterhalb der Oberflächenschicht, die man am ehesten als organischer Horizont $\left(A_{0}\right)$ bezeichnen kann, ist der Mineralboden für das Auge völlig undifferenziert. Die kleine Farbänderung in $20 \mathrm{~cm}$ Tiefe ist darauf zurückzuführen, daß oberhalb davon Wurzeln vorkommen, die tiefer völlig fehlen. Die Oberfläche des Mineralbodens (AC) ist somit als ein Übergangshorizont zu bezeichnen. Es handelt sich also um eine Rohbodenphase der Bodenentwicklung, möglicherweise eine Zwischenstufe der Podsolierung (s. SCHEFFER \& SCHACHTSCHABEL 1970, S. 323-325).

Die chemischen Analysen ergänzen nicht eindeutig die Feldbeobachtungen. Sie zeigen zwar, daß der Anteil der organischen Stoffe mit zunehmender Tiefe abnimmt und daß die Oberfläche etwas saurer ist als die tieferen Schichten. Insofern ist die Tendenz dieselbe wie in den Profilen 1 und 2. Dagegen sind die Kurven für Eisen und Aluminium anders gerichtet, als man eigentlich erwarten sollte. Die Begründung dafür mag sein: die eventuelle Eluvialschicht ist so dünn, daß die oberste Probe außer der Eluvialschicht auch die «Illuvialschicht» umfaßt.

Für alle drei Profile ist auch der Schwefelgehalt bestimmt worden (Abb. 5-7). Die vertikalen Variationen verteilen sich ähnlich wie bei Eisen und Aluminium; möglicherweise bewegt sich der Schwefel im Boden als $\mathrm{Fe}$ - und Al-Verbindung (SCHEFFER \& SCHACHTSCHABEL 1970, S. 297, 298). Es scheint auch, daß der Schwefelgehalt etwas höher ist in den A- und B-Horizonten als im Substrat, was damit zusammenhängt, daß mehr Schwefel im organischen als im anorganischen Boden vorhanden ist (s. JAUHIAINEN 1977, S. 11) und ferner auch damit, daß Schwefel den Oberflächenschichten durch Niederschlag zugeführt wird, ein Ergebnis der Luftverschmutzung durch die Industrie.

\section{SchluBfolgerungen}

In den kühl-feuchten Verhältnissen im Vorfeld des Steingletschers entwickeln sich im allgemeinen Podsolböden; infolge des Klimas ist die Humusbildung beträchtlich. Humusreiche Podsole charakterisieren auch die kalkarmen subalpinen Gebiete in der ganzen Schweiz (schmid 1961, S. 23). Auch mehrere lokale Faktoren beeinflussen die Bodenbildung. So ist z. B. im Vorfeld des Steingletschers Braunerde besonders dort anzutreffen, wo der Feinmaterialgehalt hoch und die Schneebedeckung relativ kurz ist (KING 1974, S. 32 und 35 ).

Aus den hier studierten Fällen ist der fast 10000 Jahre alte Boden (Profil 1) ein Eisenhumuspodsol mit deutlich für das Auge sichtbaren Horizonten. In 300 bis 350 Jahre altem Boden (Profil 2) sind die Podsolierungsprozesse schon deutlich im Gang, und die Horizonte sind chemisch, dagegen fast kaum mit bloßem Auge, zu unterscheiden. Nach einer Entwicklung von 120 bis 130 Jahren hat die Bodenbildung erst eine Rohbodenphase erreicht, und eine Horizontenbildung ist auch nicht durch chemische Analysen nachzuweisen (Profil 3).

Das Material dieser Untersuchung ist bescheiden und gestattet keine weitreichenden Schlüsse. Die Ergebnisse aber geben eine gewisse Einsicht in die Verhältnisse der Bodenbildung und der Bodenentwicklung vor dem Steingletscher.

\section{Danksagung}

Die Untersuchung ist durch ein Stipendium von Jenny ja Antti Vihurin rahasto unterstützt worden, wofür die Verfasser ihren besten Dank aussprechen. Herr Prof. 
Dr. Heinrich Reinhard sei für sprachliche Durchsicht des Manuskripts herzlich gedankt.

\section{Literatur:}

BURGER, RUDOLF U.HERBERT FRANZ (1969): Die Bodenbildung in der Pasterzenlandschaft. Wissenschaftliche Alpenvereinshefte 21, 253-264. München.

JAUHIAINEN, ERKKI (1977): Some trace elements of podzol profiles in southeastern Norway and western Denmark. Commentationes Biologicae, Societas Scientiarum Fennica 86.

KING, LORENZ (1974): Studien zur postglazialen Gletscher- und Vegetationsgeschichte des Sustenpaßgebietes. Basler Beiträge zur Geographie 18.

KÖSTER, ERHARD u. HARTMUT LESER (1967): Geomorphologie I: Bodenkundliche Methoden, Morphometrie und Granulometrie. Georg Westermann Verlag, Braunschweig.

Landeskarte der Schweiz 1:25000, Blatt 1211, Meiental.

Munsell, Soil Color Charts. 1954, Baltimore.

SCHEFFER,FRITZU.PAUL SCHACHTSCHABEL (1970): Lehrbuch der Bodenkunde, Siebente Auflage. Ferdinand Enke Verlag, Stuttgart.

SCHMID, EMIL (1961): Erläuterungen zur Vegetationskarte der Schweiz. Beiträge zur geobotanischen Landesaufnahme der Schweiz 39.

\section{Literaturbesprechung}

BAHRENBERG G./TAUBMANN W. (Hrsg.): Quantitative Modelle in Geographie und Raumplanung. Bremer Beiträge z.Geographie und Raumplanung, Universität Bremen 1978, 153 S.

In diesem ersten Heft der "Bremer" Beiträge sind 10 bemerkenswerte Vorträge vereinigt, die Gegenstand eines Rundgesprächs der Deutschen Forschungsgemeinschaft waren. Der Begriff Modell scheint zwar etwas weit gefasst, doch ist ja im Grunde alle Wissenschaft Modellforschung. Der Geographie engern Sinnes widmen sich die beiden Aufsätze: W. Laschinger u. L.Lötscher: Systemtheoretischer Forschungsansatz in der Humangeographie dargestellt am Beispiel von Basel und R.Hantschel: Geographie heute - zwischen Wissenschaftstheorie und Technokratie, die sich vor allem durch ihre Vorbehalte gegenüber der "'modischen" Wissenschaftstheorie auszeichnen. Die übrigen Artikel gelten mehr planerisch-geosozialen Problemen: J. Nipper und U. Streit: Mode11konzepte zur Analyse, Simulation und Prognose raumzeit-varianter stochastischer Prozesse, M.
SCHÜEPP, M. (1960): Lufttemperatur. Klimatologie der Schweiz C:1, Beiheft zu den Annalen der Schweizerischen Meteorologischen Zentralanstalt, Jahrgang 1959.

SPICHER, AUGUST (1972): Geologie. Atlas der Schweiz $1: 500000$.

UTTINGER, H. (1965): Niederschlag. Klimatologie der Schweiz E:1-3, Beiheft zu den Annalen der Schweizerischen Meteorologischen Zentralanstalt, Jahrgang 1964.

zollitsCH, B. (1969): Die Vegetationsentwicklung im Pasterzenvorfeld. Wissenschaftliche Alpenvereinshefte 21, 267-290. München.

\section{Abstract}

Soil development in high mountains: an example from the Steingletscher foreland, Switzerland.

Three soil profiles on moraines of different age were studied, and chemical analyses were made. The oldest soil (age 10000 years) an iron-humus podzol, showed distinct horizons, the second one (age 300-350 years) showed signs of beginning podzol formation; tendencies to horizons could be seen from the chemical analyses but hardly by the eye. The youngest profile (age 120-130 years) was still an undeveloped raw soil, and not even the chemical analyses showed any differentiation.
M.Fischer: Theoretische und methodische Probleme der regionalen Taxonomie, I.Schilling: Polarisationstheoretische Grundlagen einer Raumordnungskonzeption für nationale Städtesysteme, E.Giese: Weiterentwicklung und Operationalisierung der Standort- und Nutzungstheorie von ALONSO für städtische Unternehmen, W.Gaebe: Entwicklungsdynamik räumlicher Tätigkeitskomplexe, D. Höllhuber: Sozialgruppentypische Wohnstandortspräferenzen und innerstädtische Wohnstandortswahl, R.Koch: Ein Beitrag zur Weiterentwicklung regionaler Bevölkerungsprognoseansätze und V.C.Petersen: Kritik systemtheoretischer Planungsansätze. Erfreulich ist bei fast allen Beiträgen das Bemühen, der angewandten Forschung zu dienen und wie die Herausgeber G.Bahrenberg und W.Taubmann betonen - die Zusammenarbeit zwischen Geographie und Raumplanung fördern. Dem Aufsatz von Laschinger und L.Lötscher kommt dabei besondere Beachtung durch ihr schweizerisches Thema $z u$.

GH $3 / 80$
E. Winkler 